\title{
Spatial optical filter sensor based on hollow-core silica tube
}

\author{
Marta S. Ferreira, ${ }^{1,2}$ Kay Schuster, ${ }^{3}$ Jens Kobelke, ${ }^{3}$ José L. Santos, ${ }^{1,2}$ and Orlando Frazão ${ }^{1, *}$ \\ ${ }^{1}$ INESC Porto, Rua do Campo Alegre, 687, 4169-007 Porto, Portugal \\ ${ }^{2}$ Faculdade de Ciências da Universidade do Porto, Rua do Campo Alegre, 687, 4169-007 Porto, Portugal \\ ${ }^{3}$ Institute of Photonic Technology Jena, Albert-Einstein-Str. 9, 07745 Jena, Germany \\ *Corresponding author: ofrazao@inescporto.pt
}

Received December 7, 2011; revised January 20, 2012; accepted January 25, 2012; posted January 25, 2012 (Doc. ID 159591); published February 24, 2012

\begin{abstract}
A spatial optical filter based on a hollow-core silica tube is proposed. Because of the hollow-core dimensions, it is possible to obtain a periodical spatial filter ranging from 1200 to $1700 \mathrm{~nm}$ with a channel spacing of $2.64 \mathrm{THz}$. The bandwidth is approximately $5.32 \mathrm{~nm}$, and the isolation loss is $\sim 30 \mathrm{~dB}$. The optical losses are approximately $\sim 0.67 \mathrm{~dB} / \mathrm{mm}$ for a wavelength of $1500 \mathrm{~nm}$. The $40 \mathrm{~mm}$ long spatial optical filter is tested as a sensing element and subjected to different physical parameters. The spatial optical filter is wavelength sensitive to strain and temperature, while for refractive-index variations there is an optical power dependency. This fiber structure can be used as a sensing element for extreme conditions, such as in very high temperature environments, where it presents a sensitivity of $27.5 \mathrm{pm}^{\circ} \mathrm{C}^{-1}$. (C) 2012 Optical Society of America
\end{abstract}

OCIS codes: $\quad 060.0060,060.2370,120.3180$.

The development of optical fiber technology highly influenced the way optical communications and optical sensing were addressed over the years.

Besides the central utilization of the optical fiber as a high performance channel, the possibility to modify the light that travels in the fiber by the external environment, or in a controlled way, permits the buildup of the optical fiber sensing or signal processing functionalities. In the latter case, for instance it is possible to obtain a fiber device that either controls intensity or selects wavelengths, resulting into structures that act as intensity or spectral optical filters.

One example of intensity filters is the traditional optical coupler. This device is made by stretching two fibers, which originates a split in the optical power between the two output arms. [1] In respect to spectral optical filters, when it is required to mix or separate two optical signals, a wavelength division multiplexer is a widely used solution, since it provides variable wavelength selectivity and tunable central wavelength transmission [2]. These types of filters can also be implemented with integrated optics technology. For instance, spectral filters have an operation that relies on multimode interference, an approach that permits the separation of antisymmetric and symmetric modes or even the elimination of spurious unwanted modes [3].

Recently, with the arising of microstructured fibers, the production of new spectral filters became easier, just by modifying the geometry and design of the fiber. The hollow-core photonic crystal fiber (PCF) has been shown to be a good example. Its filtering behavior is due to the fact that higher order air-guided modes are characterized by very high losses, rapidly leaking from the hollow core [4]. The Bragg fiber is also another example of this type of filter. Because of its geometry, it can select a particular mode from an ensemble of modes [5]. These spectral optical filters can be used as sensors, being sensitive to physical parameters. For example, Boucouvalas et al. [6] presented the refractive-index response of biconicaltapered coaxial couplers.
Recently, different interferometric topologies of fiber sensors based on hollow-core tubes were proposed. Ferreira et al [7] presented a Fabry-Perot cavity of simple design that was sensitive to refractive-index variations without the need of a diaphragm. Furthermore, Jung et al. [8] presented an ultracompact inline broadband Mach-Zehnder interferometer, where different hollow optical fiber lengths were analyzed. Finally, a high-sensitivity broadband fiber micro-Michelson interferometer using a single-mode fiber end-spliced with a spheredend hollow-core fiber has been proposed [9].

In this Letter, a spectral optical filter with sensing properties is presented. The sensing head is based on a hollow-core silica tube. Because of the multimodal interference in the cladding of the hollow-core tube, the typical behavior of a spatial optical filter is obtained. The $40 \mathrm{~mm}$ long sensing head is characterized for strain, temperature and refractive index.

The experimental setup, shown in Fig. 1, was constituted by a supercontinuum optical source with a spectral bandwidth of $700 \mathrm{~nm}$, an optical spectrum analyzer (OSAANDO AQ6315B) with a resolution of $0.5 \mathrm{~nm}$, and a small section of hollow-core tube spliced between two sections of standard single-mode fiber (SMF28). The hollowcore tube had cladding and core diameters of 125 and $20 \mu \mathrm{m}$, respectively. In respect to the SMF28 dimensions

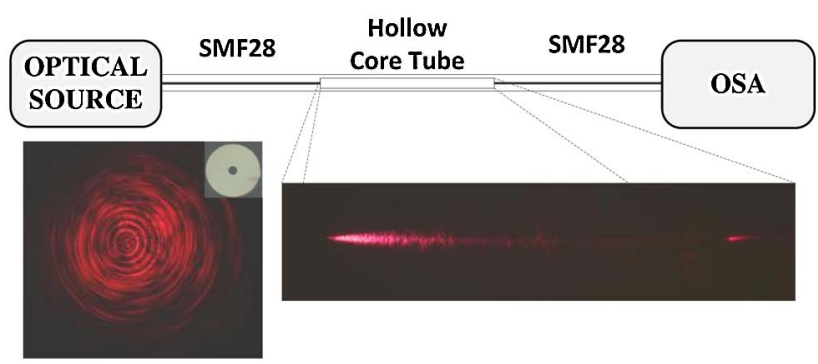

Fig. 1. (Color online) Experimental setup: Hollow-core tube cross section image and longitudinal image, both when illuminated. Inset: hollow-core tube cross section image without being illuminated. 
cladding and core diameters are 125 and $8.2 \mu \mathrm{m}$, respectively. Figure 1 also presents the cross section image when the hollow-core tube was not illuminated (inset) and when illuminated by a $680 \mathrm{~nm}$ laser. Besides, the longitudinal image of the hollow-core tube when illuminated is also exhibited. The presence of multimodal interference inside the cladding of the hollow-core tube is evidenced at the cross section image, where three concentric rings of cladding guided light can be observed, besides the different constructive and destructive interferences observed in the outer region. There is also some fraction of light traveling inside the hollow core. Regarding the longitudinal image, the cladding excitation is observed, as well as in both spliced regions. The transmission spectral response, exhibited in Fig. 2 , reveals the behavior of an optical spectral filter, as for certain wavelengths there is transmission of light (constructive interference), with a channeled behavior, whereas for other wavelengths destructive interference occurs. This filtering occurs inside the hollow-core tube, where several modes are excited. These modes will interfere and only those that are recoupled by the SMF28 contribute to the light output spectrum. Sensing heads with a length of 10 , 30, 40, and $50 \mathrm{~mm}$ were used. Even though the optical power decreased for longer sensing heads, the optical filter itself kept its light transmission properties. The decrease in optical power was expected, given that with the increase of the tube section used, the losses become higher. For wavelengths of 1300 and $1500 \mathrm{~nm}$, the optical loss is $\sim 0.55 \mathrm{~dB} / \mathrm{mm}$ and $\sim 0.67 \mathrm{~dB} / \mathrm{mm}$, respectively. It should also be noted that the splices, done in the manual program of the splicing machine, also influenced the response in optical power. The arc discharge was mainly applied to the SMF28 regions. The details on the manual program used can be consulted in [10].

The main characteristics of the spectral filter are summarized in Table 1. For comparison, in the first column the average values are presented for sensing head lengths of 10, 30, and $50 \mathrm{~mm}$. The $40 \mathrm{~mm}$ long sensing head exhibited values that did not match the remaining ones, as can be seen in the second column of Table 1 . This length presents a $\sim 2.01 \mathrm{~nm}$ reduction of the FWHM bandwidth. This difference is due to the fact that at this length all the light in the core region is in phase. When compared with a multimode interferometer, the self-imaging occurs at a similar length with a maximum coupled efficiency of

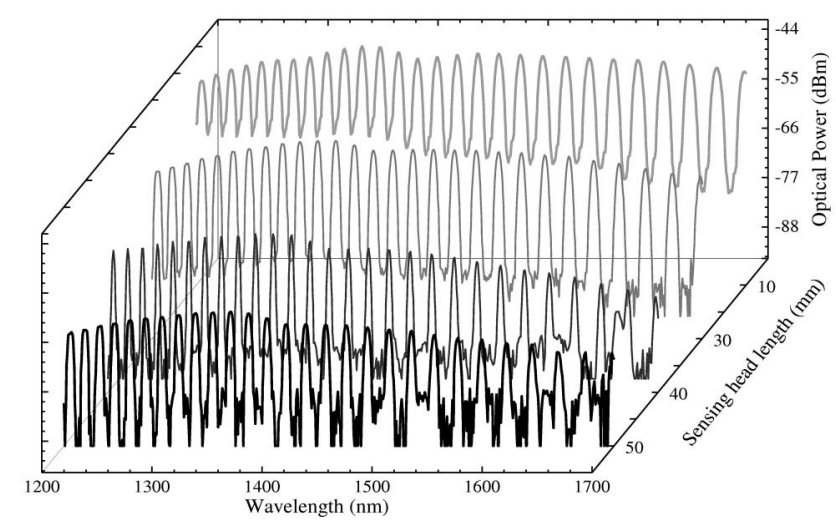

Fig. 2. Transmission spectral response for sensing head lengths of 10, 30, 40, and $50 \mathrm{~mm}$.
Table 1. Spatial Optical Filter Properties

\begin{tabular}{|c|c|c|}
\hline & Average Value & $L=40 \mathrm{~mm}$ \\
\hline FWHM (nm) & 5.32 & 3.30 \\
\hline Channel Spacing (THz) & 2.65 & 2.68 \\
\hline Channel Pass-band (THz) & 1.13 & 4.86 \\
\hline Channel Band-reject (THz) & 1.54 & 1.98 \\
\hline Adjacent Channel Crosstalk (dB) & 28.58 & 28.58 \\
\hline
\end{tabular}

light. The $40 \mathrm{~mm}$ long sensing head was characterized in strain, temperature, and refractive index. In relation to strain measurements, done at room temperature, the sensing head was attached to a translation stage with a resolution of $1 \mu \mathrm{m}$. Figure 3 shows the wavelength dependence with strain variation.

The strain sensitivity obtained was of $-0.59 \mathrm{pm} \cdot \mu \varepsilon^{-1}$. The negative response is due to the dominance of the silica thermo-optic effect. It must be highlighted that the spectrum only varies in wavelength, the remaining characteristics, such as FWHM and channel spacing, are preserved. Regarding temperature measurement, the sensing head was placed inside a tubular oven, and the temperature reading had a resolution of $0.1{ }^{\circ} \mathrm{C}$. The results obtained are presented in Fig. 4 . The response to temperature can be well adjusted by the second order polynomial

$$
\Delta \lambda=7.52 \times 10^{-6} \mathrm{~T}^{2}+0.017 \mathrm{~T}-0.63 .
$$

Given the positive slope the thermal expansion is the dominant effect. An analysis of the data in Fig. 4 indicates the feasibility of separating the temperature range in two intervals (insets of the figure), in each of them exhibiting the structure a fairly linear behavior, with sensitivities of $20.6 \mathrm{pm}^{\circ} \mathrm{C}^{-1}$ (in the temperature range up to $400{ }^{\circ} \mathrm{C}$ ) and $27.5 \mathrm{pm}^{\circ} \mathrm{C}^{-1}$ (in the interval $450{ }^{\circ} \mathrm{C}$ to $900{ }^{\circ} \mathrm{C}$ ). The FWHM of the transmission spectral windows and the channel spacing changed slightly, $\sim 0.17 \mathrm{~nm}$ and $\sim 0.30 \mathrm{~nm}$, respectively, in a temperature variation of $500{ }^{\circ} \mathrm{C}$. Finally, refractive index measurements were done by submerging the sensing head in several liquids

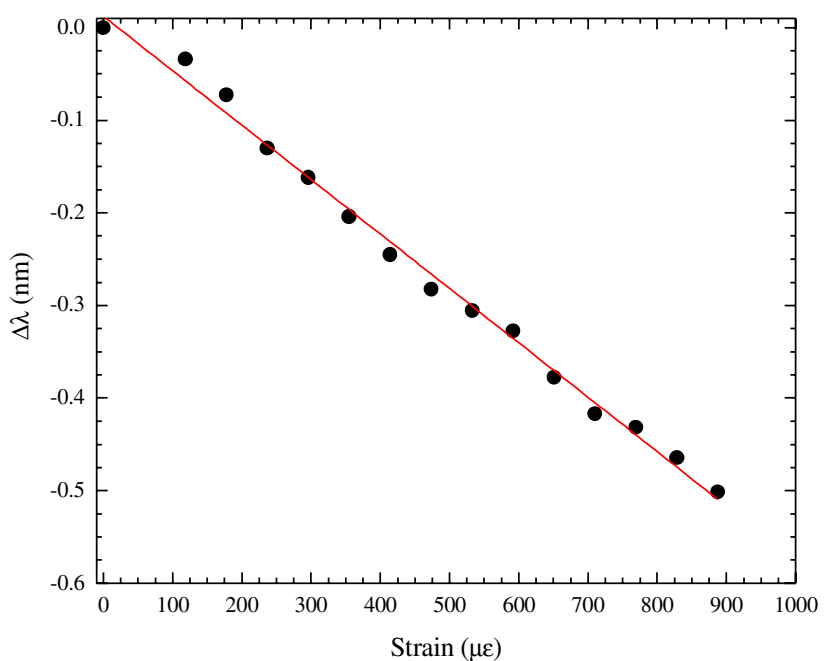

Fig. 3. (Color online) Sensing head response to the applied strain. 


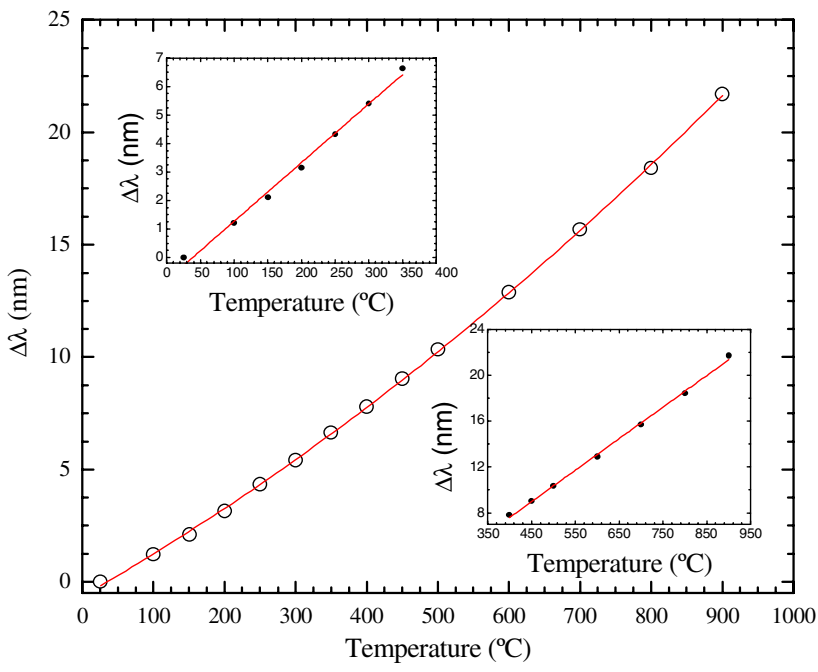

Fig. 4. (Color online) Temperature response of the sensing head. Inset 1 (top left): low temperatures response; inset 2 (bottom right): high temperatures response.

with different refractive index. As evidenced in Fig. $\underline{5}$, there is no wavelength shift, only a decrease in the optical power as the refractive index increases. This is a mirror-like behavior. When immersed in a liquid with a refractive index close to silica, the optical filter disappears, as expected, since there is radiation propagation outward from the hollow-core tube. It was also verified that these measurements must be done away from the splices region; otherwise large losses are present.

Summarizing, a spectral optical filter based on a hollow core silica tube was proposed. Several filters with different lengths were fabricated and their spectral characteristics studied. A $40 \mathrm{~mm}$ long filter was tested as a sensing element, being subjected to strain, temperature, and refractive index variations. It exhibited wavelength sensitivity for the first two parameters, and optical power variations for the last one. The wavelength sensitivities obtained were of $-0.59 \mathrm{pm} \cdot \mu \varepsilon^{-1}$ for strain and $20.6 \mathrm{pm}^{\circ} \mathrm{C}^{-1}$ and $27.5 \mathrm{pm}^{\circ} \mathrm{C}^{-1}$ for the low and high temperature ranges, respectively. This structure is easy to fabricate and quite compact, and the results are highly reproducible. Besides use as a sensing element, the employment of this spatial filter to interrogate other types of

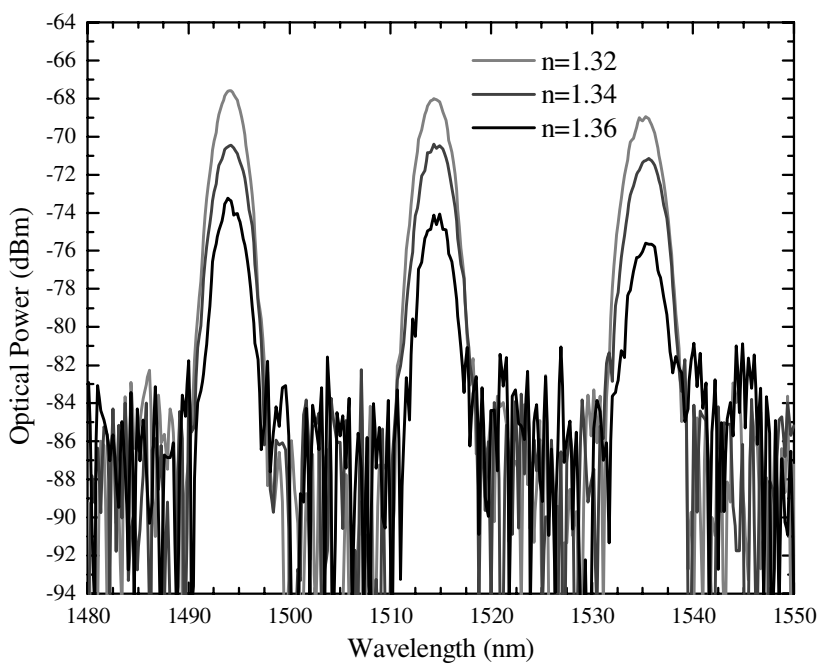

Fig. 5. Sensing head spectra for different refractive index media.

sensors, by filtering the signals, is also an attractive solution. This is a work in progress.

\section{References}

1. B. S. Kawasaki, K. O. Hill, and R. G. Lamont, Opt. Lett. 6, 327 (1981).

2. C. M. Lawson, P. M. Kopera, T. Y. Hsu, and V. J. Tekippe, Electron. Lett. 20, 963 (1984).

3. J. Leuthold, R. Hess, J. Eckner, P. A. Besse, and H. Melchior, Opt. Lett. 21, 836 (1996).

4. S. O. Konorov, V. P. Mitrokhin, A. B. Fedotov, D. A. SidorovBiryukov, V. I. Beloglazov, N. B. Skibina, E. Wintner, M. Scalora, and A. M. Zheltikov, Phys. Med. Biol. 49, 1359 (2004).

5. M. E. Likhachev, S. L. Semjonov, M. M. Bubnov, E. M. Dianov, V. F. Khopin, M. Yu Salganskii, M. A. Gurjanov, A. N. Gurjanov, R. Jamier, P. Viale, S. Fevrier, and J.-M. Blondy, Quantum Electron. 36, 581 (2006).

6. A. C. Boucouvalas and G. Georgiou, Opt. Lett. 11, 257 (1986).

7. M. S. Ferreira, L. Coelho, K. Schuster, J. Kobelke, J. L. Santos, and O. Frazão, Opt. Lett. 36, 4029 (2011).

8. Y. Jung, S. Lee, B. H. Lee, and K. Oh, Opt. Lett. 33, 2934 (2008).

9. N. Chen, K. Lu, J. Shy, and C. Lin, Opt. Lett. 36, 2074 (2011).

10. O. Frazão, J. P. Carvalho, and H. M. Salgado, Microw. Opt. Technol. Lett. 46, 172 (2005). 